\section{Research in Oncology: A new start}

Research in Oncology journal continues the mission of Kasr El-Aini Journal of Clinical Oncology and Nuclear Medicine which was established in 2005 by Kasr El-Aini Center of Clinical Oncology and Nuclear Medicine - Faculty of Medicine - Cairo University as one of the few peer-reviewed journals specialized in oncology in our region.

Research in Oncology aims at publishing high quality research in all oncology fields including, but not limited to, prevention, early detection, diagnosis, treatment and supportive and palliative care. Researchers from all disciplines working in the oncology field are invited to submit their manuscripts to Research in Oncology.

The changes in the journal are not only in the title, but also include many other new features. Among these, is the online submission through an Electronic Editorial System (EES) under the URL (http://resoncol. js.iknito.com/) to facilitate the submission and review of manuscripts. Authenticity of submitted manuscripts is cross-checked using professional plagiarism-detection software. In addition, accepted manuscripts undergo professional technical and language editing.
Research in Oncology continues an open access policy to ensure easier accessibility to published articles. Once an accepted article is ready for publish, it will appear online as "article in press" with digital object identifier (DOI) assignment. Thereafter, articles in press are published in a journal issue in both online and print formats.

For wider dissemination of knowledge, the editorial team is currently working on indexing Research in Oncology in major scientific research databases.

Understanding the importance of the time factor for authors, efforts will be done to assure the shortest time possible from submission to decision and to expedite the publishing process of accepted manuscripts.

The editorial staff of Research in Oncology is indebted to the previous editorial members of Kasr ElAini Journal of Clinical Oncology and Nuclear Medicine and would like to thank all of them for their efforts in the last eleven years. 